University of Wollongong

Research Online

Faculty of Engineering and Information

Faculty of Engineering and Information

Sciences - Papers: Part A

Sciences

$1-1-2014$

\title{
An advanced mathematical model and its experimental verification for trilayer conjugated polymer actuators
}

Chuc Nguyen

University of Wollongong, chuc@uow.edu.au

Gursel Alici

University of Wollongong, gursel@uow.edu.au

Gordon G. Wallace

University of Wollongong, gwallace@uow.edu.au

Follow this and additional works at: https://ro.uow.edu.au/eispapers

Part of the Engineering Commons, and the Science and Technology Studies Commons

Research Online is the open access institutional repository for the University of Wollongong. For further information contact the UOW Library: research-pubs@uow.edu.au 


\title{
An advanced mathematical model and its experimental verification for trilayer conjugated polymer actuators
}

\begin{abstract}
This paper describes the establishment of an enhanced mathematical model and an inversion-based controller based on the proposed model for a trilayer conjugated polymer actuator that will steer a cochlear implant through a 3-D structure. The multilayer electroactive polymer actuator that operates in air will suit many biomedical applications. We propose to use viscoelastic models for the conducting polymer and membrane layers of the actuator so that its mechanical properties can be incorporated into the actuator more accurately. The proposed model accurately predicts the frequency response of the electrical admittance and curvature of the conjugated polymer actuators, and its efficacy for different actuators has been experimentally evaluated. In addition, an inversion-based controller without an external sensor for position feedback data has successfully been evaluated to further validate the ability of the proposed model for sensorless position control of the actuators.
\end{abstract}

\section{Keywords}

model, its, verification, experimental, trilayer, advanced, conjugated, polymer, actuators, mathematical

\section{Disciplines}

Engineering | Science and Technology Studies

\section{Publication Details}

Nguyen, C. Huu., Alici, G. \& Wallace, G. (2014). An advanced mathematical model and its experimental verification for trilayer conjugated polymer actuators. IEEE-ASME Transactions on Mechatronics, 19 (4), 1279-1288. 


\title{
An Advanced Mathematical Model and its Experimental Verification for Tri-layer Conjugated Polymer Actuators
}

\author{
Chuc Huu Nguyen, Gursel Alici and Gordon Wallace
}

\begin{abstract}
This paper describes the establishment of an enhanced mathematical model and an inversion-based controller based on the proposed model for a tri-layer conjugated polymer actuator that will steer a cochlear implant through a 3D structure. The multi-layer electroactive polymer actuator that operates in air will suit many biomedical applications. We propose to use viscoelastic models for the conducting polymer and membrane layers of the actuator so that its mechanical properties can be incorporated into the actuator more accurately. The proposed model accurately predicts the frequency response of the electrical admittance and curvature of the conjugated polymer actuators, and its efficacy for different actuators has been experimentally evaluated. In addition, an inversion-based controller without an external sensor for position feedback data has successfully been evaluated to further validate the ability of the proposed model for sensorless position control of the actuators.
\end{abstract}

Index Terms - actuators, electroactive polymer actuators, inversion-based feedforward control

\section{INTRODUCTION}

$\mathrm{T}$ The past decade has seen the rapid development of electroactive polymers (EAPs) as smart materials to establish innovative actuation and sensing concepts [1]. These tri-layer conjugated polymer actuators [2, 3], which are dry EAP actuators, can operate under a low voltage in both dry and wet environments, unlike other ionic-EAP actuators. This class of actuators generates bending displacements under an electrical potential difference. This simple operating principle is based on the conversion of electrochemical energy to mechanical energy and it offers new horizons for the cutting-edge applications typified by biomedical applications [2]. An electro-chemo-mechanical model of the actuators must be obtained in order to understand their dynamic behaviour and the control strategies needed to improve their ability to follow a useruser-specified input. As an example, we propose to use dry actuators to guide a cochlear implant through a $3 \mathrm{D}$ structure, much like an implant carrier [4].

*Research supported by ARC Centre of Excellence for Electromaterials Science (Grant No. CE0561616)

C. H. Nguyen and G. Alici are with the School of Mechanical, Materials and Mechatronic Engineering and ARC Centre of Excellence for Electromaterials, University of Wollongong, Australia (e-mail: chuc@) uow.edu.au, gursel@uow.edu.au).Corresponding author: G. Alici. G. G. Wallace is with ARC Centre of Excellence for Electromaterials, University of Wollongong, Australia (e-mail: gwallace@uow.edu.au)
Such intricate medical applications require actuators with built-in sensors or control algorithms that do not need external sensing data for accurate positioning. For the cochlear implant application that we consider here, for example, it is virtually impossible to implement sensory control and this means there is an increasing need to use inversion control methods based on an accurate electroelectro-chemo-mechanical model of the actuator [2].

Some of the previous work on the dynamic model of this actuator includes Madden [5] who established a diffusiveelastic-metal model for a bi-layer conducting polymer actuator. This model has been extended to a tri-layer EAP actuator by Fang et al. [6]. The diffusive impedance in this model is assumed to be connected with a double layer capacitor in parallel [5], while Shoa et al. [7] developed an equivalent transmission line model for bi-layer and tri-layer actuators. Their model describes the time response of the current as well as the tip displacement of the actuator. All the models in previous research considered that the capacitance of the conducting polymer layer was connected with the diffusion impedance in parallel, but in this paper we developed a new model where the capacitance of the conducting polymer layer was connected in series. The structure of our model agrees with a previous finding on a more accurate estimation of actuator impedance, admittance, and Bode plots from an equivalent electrical circuit model where both impedance and capacitance are connected in series $[9,14]$. It must be noted that the elastic modulus of the actuator is not constant during its operation [8]. Indeed, Nguyen et al. [9] have reported that the chargeto-strain ratio varies with the applied voltage. By assuming that this ratio is constant, however, we have developed viscoelastic models for the conducting polymer and membrane layers of the actuators to increase the accuracy of the actuator model. The main contribution of this paper is that it establishes and experimentally validates a comprehensive and accurate electromechanical model describing the dynamic behaviour of the actuators for a wider range of frequencies. We used a non-linear least square estimation method to identify the numerical values of the model parameters from the experimental results.

Another difficulty is that the use of control theories on feedback sensors to control a tri-layer conducting actuator has some disadvantages due to the size of sensor. an example, although Fang et al. [6] successfully implemented a robust adaptive controller based on an electrochemical model using a laser sensor for displacement feedback, in real applications it is not practical to apply a control algorithm that requires external feedback where there is no space for an external feedback sensor. The use of feedback sensors is a disadvantage with the proposed 
actuators, particularly in the biomedical applications we are developing where space is of paramount importance. As stated above, to overcome the limitations of using external sensors, it is better to use an inversion-based feedforward controller to control the position of this actuator because it based on inverting a valid mathematical model to calculate the control input needed to generate the desired displacement. Previously, a black box model of the tri-layer conducting polymer actuator was developed for its inversion-based position control [10]. This model was developed for a particular actuator and when the size and other properties were changed, a new model that could accurately control the position of the actuator had to be identified. Unlike previously published models, in this we went a step beyond our previous work to establish an inversion controller based on an enhanced electro-chemoelectro-chemo-mechanical model of the actuator. The experimental results from the implementation of this inversion-based position control showed the validity of the proposed model.

The remainder of this paper is organised as follows: in Section II we introduce the proposed model, in Section III we provide an experimental verification of the proposed model; the experimental results from the inversion based controller are presented in Section IV and the conclusions and future work are outlined in Section V.

|

\section{THE PROPOSED MODEL OF A TRI-LAYER ACTUATOR}

A. The operating principle of the tri-layer conducting polymer actuator

I Fig. 1Fig. 1 shows the structure of the tri-layer actuator which consists of three main layers: two polypyrrole (PPy) layers that are active components and an inner porous separator of polyvineylindence fluoride (PVDF) that holds the liquid electrolyte and the lithium trifouromethanesulfonimide $\left(\mathrm{Li}^{+} \mathrm{TFSI}\right)$ salt in the solvent of propylene carbonate $(\mathrm{PC})$. The whole structure is like a cantilever beam with one end fixed and the other end free. It has a large amplitude bending motion when a voltage is applied across the two PPy layers that act as electrodes, as | shown in Fig. 2Fig. 2.

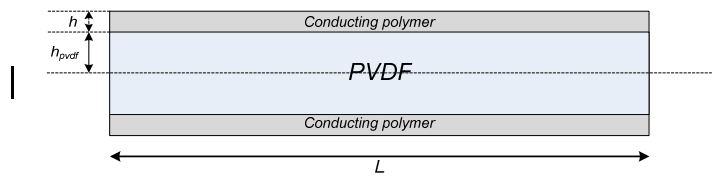

Fig. 1. The schematic of trilayer conducting polymer actuator
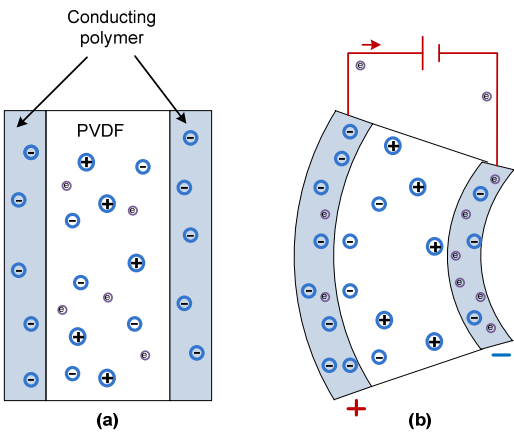

$\oplus$ cation

$\odot$ anion

- electron

Fig. 2. The mechanism of a tri-layer conducting polymer actuator. (a) Cross sectional view of actuators (b) Bending of actuator when a voltage is applied

The operating principle of the tri-layer conducting polymer actuators is described in detail in our previous papers $[3,11,12]$. When a voltage passes between the two conducting polymer layers (PPy) of the actuator, as shown in Fig. 2Fig. 2, one conducting polymer layer is oxidised while the other is reduced. The oxidised layer takes in anions and expands [2], while the reduced layer contracts because the anions leave this layer. The overall result is that this cantilevered structure will bend towards the negative electrode/cathode, as depicted in Fig. 2Fig. 2. The volume changes because the charge balancing anions move in and out of the polymer layers, and perhaps some solvent molecules move inside the polymer layers due to osmotic effects to balance the ionic concentration. The charge transfer between the anode and cathode determines the impedance of the actuator as well as the change in volume.

\section{B. The diffusive impedance of a conducting polymer} actuator

Recently, significant effort has been made to model the diffusive impedance of the actuators [14]. Madden [5], developed a diffusive model to describe the impedance of the film-type conducting polymer actuators operating in an aqueous medium. This model has been extended to a trilayer conducting polymer actuator as a double layer capacitor and a diffusive impedance element connected in parlatith f6ferazhce to [14], the equivalent electrical circuit for the conducting polymer layer is shown in Fig. 3 Fig. 3. $Z_{D}$ is the diffusion impedance and $C_{d}$ is the capacitance of the conducting polymer layer. The $Z_{D}$ element is given by:

$$
Z_{D}(s)=\frac{h}{C_{d}} \frac{\operatorname{coth}\left(h \sqrt{\frac{s}{D}}\right)}{\sqrt{D s}}
$$

where $D$ is the diffusion coefficient and $h$ is the thickness of the conducting polymer layer, as shown in Fig. 1Fig. 1. 


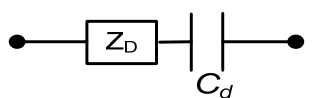

Fig. 3. Equivalent circuit for the conducting polymer layer.

C. The comprehensive model of a tri-layer conducting polymer actuator

Electrical model

The diffusive impedance model contains the electrical parameters of the actuator. When a positive voltage is applied to the PPy layer, the anions (TFSI) in the PVDF layer move towards this layer. For this reason, we have modelled, an element of PPy/PVDF layers by combining diffusive impedance $Z_{D}$ and the capacitance $\left(C_{d}\right)$, as shown Fig. 4. If we call the electronic resistance of one element of the PPy layer $R_{e}$ and that of one element of the PVDF layer $R_{p}$, we will have a transmission line circuit of the tri-layer conducting polymer actuator (PPy/PVDF/PPy), as shown in

Fig. 5Fig. 5. Assuming that the tri-layer actuator consists of an n-element of impedance with respect to its length, the | impedance of the $i$-element $Z_{i}(s)$ described in Fig. 4Fig. 4, is calculated as follows:

$$
Z_{i}(s)=R_{p}+2\left(Z_{D}+\frac{1}{s C_{d}}\right)
$$

The total impedance of the tri-layer actuator is obtained as [15]:

$$
Z(s)=\sqrt{2 R_{e} Z_{i}(s)} \operatorname{coth}\left(\sqrt{\frac{2 R_{e}}{Z_{i}(s)}} L\right)
$$

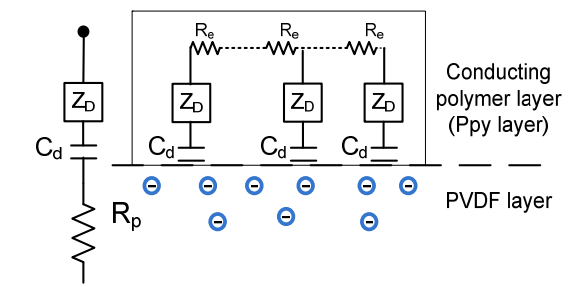

Fig. 4. The right drawing is the interface between the conducting polymer layer and the PVDF layer. The left drawing is the equivalent electrical circuit corresponding to each interface.

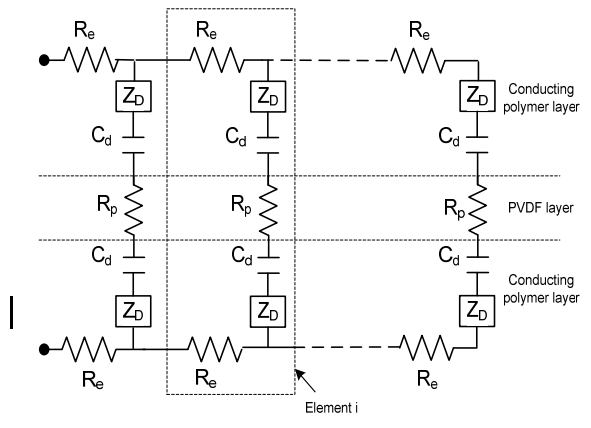

Fig. 5. The equivalent circuit of the trilayer conducting polymer actuator

\section{Mechanical model}

The strain in this actuator is assumed to be linearly proportional to the exchanged charge density [16];

$$
\varepsilon=\rho_{P P y} \cdot \alpha
$$

where $\alpha$ is the strain-to-charge density ratio and $\rho_{P P y}$ is the charge density.

The charge density of the actuator is given by:

$$
\rho_{P P y}(s)=\frac{I(s)}{s W L h}
$$

where $W$ and $L$ are the width and length of the PPy layer, respectively.

With reference to [17] and Eq. (5), the beam curvature

$(\kappa)$ is given by:

$$
\kappa=\frac{3 \alpha}{2 h_{P V D F}} \frac{\left(1+\frac{h}{h_{P V D F}}\right)^{2}-1}{\left(1+\frac{h}{h_{P V D F}}\right)^{3}+\frac{E_{P V D F}}{E_{P P y}}-1} \cdot \rho_{P P y}
$$

where $E_{P P y}$ and $E_{P V D F}$ are the elastic moduli of the polypyrrole and PVDF layers, respectively. $h$ and $h_{P V D F}$, as shown in Fig. 1Fig. 1, are the thicknesses of the PPy layer and the PVDF layer, respectively.

In addition, the elastic modulus of the PPy layer, which changes during the redox operation of the actuator, is a function of the change in the doping level [8]. When the magnitude of the applied voltage is increased, the doping level increases accordingly. Consequently, the elastic modulus of the conducting polymer changes as a function of the applied voltage. To accurately reflect this change in the elastic modulus in the proposed actuator model, a viscoelastic model of the PPy layer is used. In this paper, Kelvin-Voigt model, which was originally used to model viscoelastic behaviour of polymeric materials [18], is used here, as shown in Fig. 6Fig. 6.

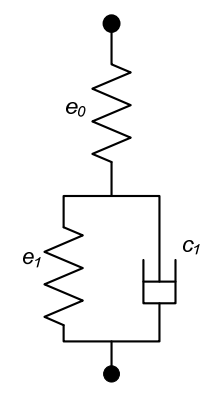

Fig. 6. Standard linear solid (SLS) model for the PPy layer [18].

From Fig. 6Fig. 6 the effective modulus of elasticity of the PPy layer is obtained by:

$$
\frac{1}{E_{P P y}}=\frac{1}{e_{0}}+\frac{1}{e_{1}+c_{1} s}
$$

Formatted: Font: $10 \mathrm{pt}$

Formatted: Font: $10 \mathrm{pt}$

Formatted: Font: $10 \mathrm{pt}$ 
where $e_{0}$ indicates the modulus of elasticity of the PPy layers under an instantaneous strain, $e_{1}$ and $c_{1}$ are the modulus of elasticity and viscosity of the PPy layers due to the maximum retarded strain and the rate of strain after the instantaneous strain region in the creep curve .

This model represents creep, hysteresis and the stress relaxation behaviour of the PPy layers [19, 20] which show the specific characteristics of viscoelastic materials. $e_{0}$ can be estimated directly from the frequency response of the actuators with no consideration of its creep behaviour [21]. From Eq. (7), the complex modulus of the PPy layers, which shows the dynamic behaviour of the PPy layers in the frequency domain, is obtained as $[19,20]$

$$
\begin{aligned}
E_{P P y}(j \omega) & =\frac{e_{0}\left(e_{1}+j \omega c_{1}\right)}{e_{0}+e_{1}+j \omega c_{1}} \\
& =E_{d}(\omega)+j E_{l}(\omega)=E_{d}(\omega)(1+j \eta(\omega))
\end{aligned}
$$

where $E_{d}(\omega), E_{l}(\omega)$ and $\eta(\omega)$ are the dynamic modulus of elasticity, and the loss modulus and loss factor, respectively. With reference to Fig. 12, while the dynamic modulus increases with the frequency, the loss modulus decreases with the frequency after passing at least one peak.

By rewriting Eq. (8), we have the following equation:

$$
\frac{1}{E_{P P y}}=\frac{1}{e_{0}}\left(1+\frac{1}{e_{1} / e_{0}+c_{1} / e_{0} s}\right)=\frac{1}{e_{0}}\left(1+\frac{1}{k_{0}+k_{1} s}\right)
$$

where $k_{0}$ and $k_{l}$ are the parameters that need to be identified. Similarly, the viscoelastic model of the PVDF layer, a kind of porous membrane [22], is described as a Kelvin-Voigt element [18], as depicted in Fig. 7. The reason why it is not represented by the model in Fig. 6, is because it is a passive layer with no active contribution to the actuation. There is no instantaneous strain in the strain curve leading to the extra modulus of elasticity in Fig. 6. This strain equation was obtained by taking the inverse Laplace transformation of Eq. (9)

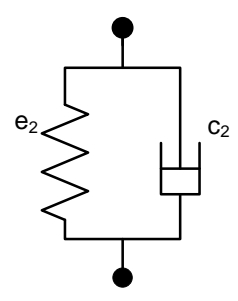

Fig. 7. The viscoelastic model of the PVDF layer.
From Fig. 7, the effective modulus of elasticity of the PVDF layer under a constant bending stress is derived as follows:

$$
E_{P V D F}=e_{2}+c_{2} s=e_{2}\left(1+\frac{c_{2}}{e_{2}} s\right)=e_{2}\left(1+k_{2} s\right)
$$

where $k_{2}$ is the parameter that must be identified. $e_{2}$ is the elastic modulus of the PVDF layer in a steady state and it can be measured directly [21]. Similar to Eq. (8), the complex modulus of the PVDF layer in the frequency domain is obtained as;

$$
E_{P V D F}(j \omega)=e_{2}+j \omega c_{2}=E_{d 2}(\omega)+j E_{l 2}(\omega)(11)
$$

where $E_{d 2}(\omega)$ and $E_{l 2}(\omega)$ are the dynamic modulus of elasticity and the loss modulus, respectively.

Based on Eqs. (6), (9) and (10), the equation can be rewritten as follows:

$$
\kappa=\frac{3 \alpha}{2 h_{P V D F}} \frac{\left(1+\frac{h}{h_{P V D F}}\right)^{2}-1}{\left(1+\frac{h}{h_{P V D F}}\right)^{3}+\frac{e_{2}\left(1+k_{2} s\right)\left(1+k_{0}+k_{1} s\right)}{e_{0}\left(k_{0}+k_{1} s\right)}-1} \cdot \rho_{P P_{y}}
$$

The displacement of the actuator was measured by a laser sensor, as shown in Fig.8Fig.8 (a). The relationship between the displacement $y_{d}=y_{0}-y$ and the curvature of the actuator is [23]

$$
\kappa=\frac{1}{r}=\frac{2 y_{d}}{y_{d}{ }^{2}+L_{m}{ }^{2}}
$$

where $r$ is the radius of curvature of the actuator upon activation.

By combining Eqs. (3) and (12) we obtain a transfer function which describes the relationship between the curvature of the actuator and an applied voltage:

$$
\frac{\kappa(s)}{V(s)}=K_{c} \frac{\tanh \left(\sqrt{\frac{2 R_{e}}{Z_{i}(s)}} L\right)}{s \sqrt{2 R_{e} Z_{i}(s)}}
$$

where

$$
K_{c}=\frac{3 \alpha}{2 h_{P V D F} h W L} \frac{\left(1+\frac{h}{h_{P V D F}}\right)^{2}-1}{\left(1+\frac{h}{h_{P V D F}}\right)^{3}+\frac{e_{2}\left(1+k_{2} s\right)\left(1+k_{0}+k_{1} s\right)}{e_{0}\left(k_{0}+k_{1} s\right)}-1}
$$

Based on Eq. (14) we can estimate the curvature of the polymer actuator under a voltage applied across its two PPy layers. 


\section{EXPERIMENTAL SETUP AND MODEL VALIDATION}

The fabrication and synthesis of the tri-layer conducting polymer actuator can be found in our previous work [3]. The PVDF was a commercial membrane with a thickness of $110 \mu m$ while the PPy was grown in 12 hours to a thickness of approximately $30 \mu \mathrm{m}$.
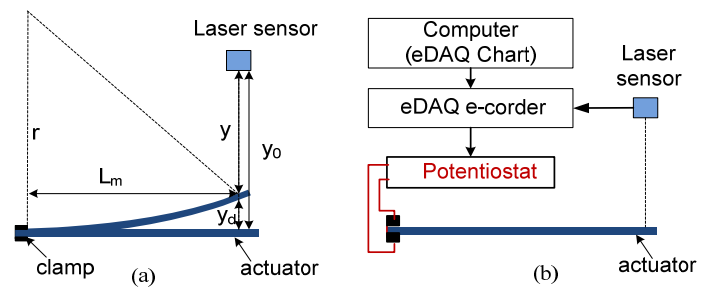

Fig.8. The schematic of measurement (a) and experimental setup (b)

To measure the bending movement of the actuator, the eDAQ e-corder recorder together with the eDAQ chart software, both supplied by eDAQ Inc, was used. The | experimental setup is shown in Fig.8Fig.8 (b). In this work, the tip displacement of the actuator was measured with a laser sensor, and then the voltage increased from $0.1 \mathrm{~V}$ to $0.5 \mathrm{~V}$ to ensure that any deflection of the tri-layer actuator was in the measuring range of the laser sensor. To develop a model for a larger deflection, we will combine the model proposed here with a non-linear mechanical displacement moded [pat]ameters of actuators such as $R_{e}$ and $R_{p}$ in Eq.

| Error! Reference source not found. can be obtained using a four point probe instrument as a measurement device. The conductivity of PPy/LiTFSI when it is wetted in the electrolyte (LiTFSI/PC) was measured as $\sigma_{e}=125 \mathrm{~S} / \mathrm{cm}$ and the conductivity of the PVDF with gold particles spattered on it and the electrolyte (LiTFSI/PC) in it was measured as $\sigma_{p}=8.85 \mathrm{~S} / \mathrm{cm}$ [25]. In our proposed model, the conductivities of the PVDF and the conducting polymer layers are assumed to be constant with respect to the dimensions of the actuator and during the oxidation state.

The resistances of the $i$-element in Fig. 5 are calculated from [25]:

$$
\begin{aligned}
& R_{p}=\frac{1}{\sigma_{p}} \frac{2 h_{P V D F}}{W} \\
& R_{e}=\frac{1}{\sigma_{e}} \frac{1}{W \cdot h}
\end{aligned}
$$

We assumed that $\sigma_{e}$ and $\sigma_{p}$ are constant parameters, however the diffusion coefficient (D) and the total capacitance $\left(C_{d}\right)$ are difficult to measure directly, although we can estimate their numerical values by comparing the experimental and numerical electrical admittance spectrums (Eq.(3)) of the actuator. Similarly, the strain-to-charge density ratio $\alpha$ and the parameters $k_{0}, k_{1}$ and $k_{2}$ in Eq. (14) could be estimated by comparing the frequency of the curvature of the tri-layer actuator with the results of the modelling. There are six parameters that must be estimated using the non-linear least square method, and this can be done by comparing the experimental results with the simulation results of Eqs. (3) and (14).

The tri-layer actuator used in our experiments is $10 \mathrm{~mm} \times 3 \mathrm{~mm} \times 0.17 \mathrm{~mm}$. The constant numerical values of elastic moduli were taken as $e_{o}=200 \mathrm{MPa}$ (PPy layer ) and $e_{2}=117 \mathrm{MPa}$ (PVDF layer) from our previously reported work [21]. The distance $L_{m}$ for all the actuators was fixed at $8 \mathrm{~mm}$ from the fixed end of the actuator. All the experiments were conducted under the same conditions, after which the actuator was kept in a solution (LiTFSI/PC) to ensure that the properties of the material did not change.

Based on an estimation of the frequency of Eq. (3) and electrical admittance spectrum, the parameters $D$ and $C_{d}$ were identified. In this paper, we assume that these parameters are constant. The experimental and simulation results are illustrated in Fig. 9Fig. 9. The modelling results give a good estimation of the proposed model compared to the experimental electrical admittance spectrum of the actuator when the voltage was changed from $0.1 \mathrm{~V}$ to $0.5 \mathrm{~V}$. Based on this estimation, the numerical values of $D$ and $C_{d}$ were identified and are shown in Table 1. The identified diffusion coefficient is within an acceptable range of the diffusion coefficients [30], which depend on the type of dopant ion, the solvent and the redox state of PPy.

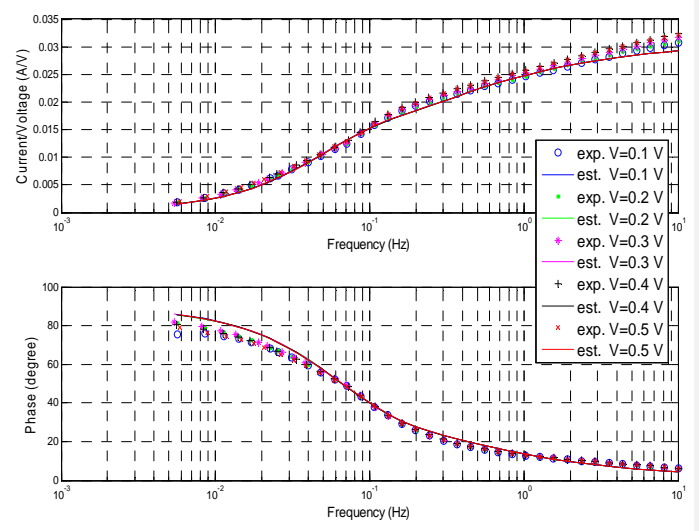

Fig. 9. Experimental and theoretical frequency responses of the electrical admittance spectra under different voltages.

Table 1. Identified parameters

\begin{tabular}{|c|c|}
\hline$I V . \quad$ Parameters & V. Value \\
\hline VI. $D\left(\mathrm{~m}^{2} / \mathrm{s}\right)$ & VII. $1.8817 \times 10^{-10}$ \\
\hline VIII. $C_{d}(\mathrm{~F} / \mathrm{m})$ & IX. 11.0591 \\
\hline$X . \quad \alpha\left(\mathrm{m}^{3} / \mathrm{C}\right)$ & XI. $1.469 \times 10^{-9}$ \\
\hline
\end{tabular}


The experimental and theoretical frequency of the | curvature are shown in Fig. 10Fig. 10, and based on this experiment, the strain-to-charge density ratio $(\alpha), k_{0}, k_{l}$ and were identified by comparing the frequency of Eq. (14) with the results of the experimental curvature. In this paper, the strain-to-charge ratio is assumed to be constant and was estimated as shown in Table 1. By matching the experimental results with the proposed model, the variations of $k_{0}, k_{1}$ and $k_{2}$ with the applied voltage are shown in

| Fig.11Fig.11. These variations can be explained by the fact that when the voltage increased, the ions moved in and out the PPy and PVDF layers [8]. These variations in the of the elasticity of the PPy are depicted in Fig.12Fig.12, where the moduli of the PPy depend on the frequency and amplitude of the applied voltage. Variations of the elastic modulus of the PPy layer are very clear at low frequencies.

When the results of the displacement response are compared with the dynamic response of the previous model [26], the proposed model can predict that the phase of displacement response will be less than $-90^{\circ}$ (i.e. $-140^{\circ}$ ). When we look at the phase of the previous model [26], the minimum phase angle of the displacement is $-90^{\circ}$, a value that does not agree with our experimental results when we increased the frequency of the voltage to $10 \mathrm{~Hz}$, as shown in | Fig. 10Fig. 10.
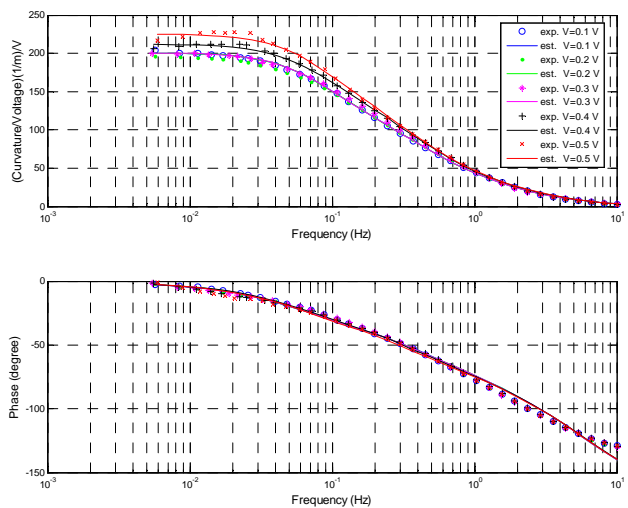

Fig. 10. Experimental and theoretical frequency responses of the actuator curvature under different voltages

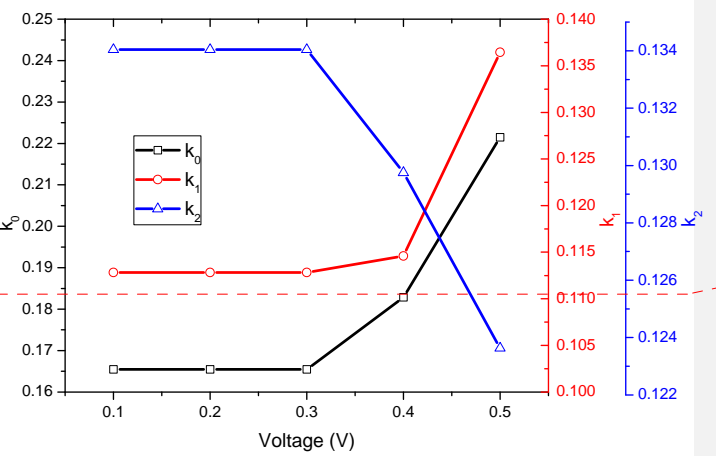

Formatted: Font: $10 \mathrm{pt}$

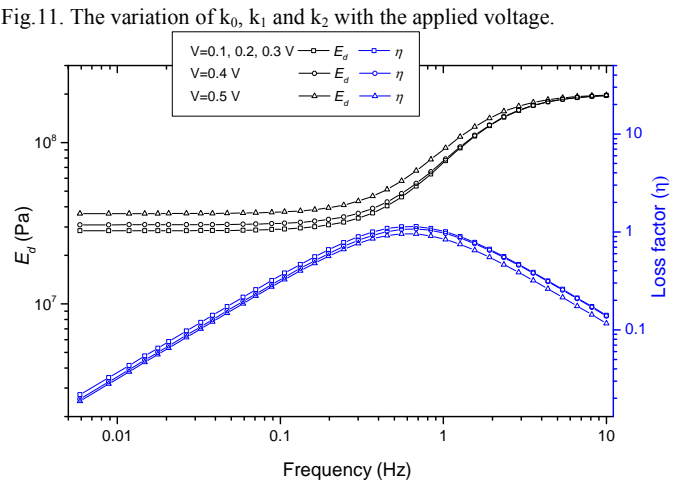

Fig.12. The variation of the moduli of the PPy layer calculated from Eq.(8)
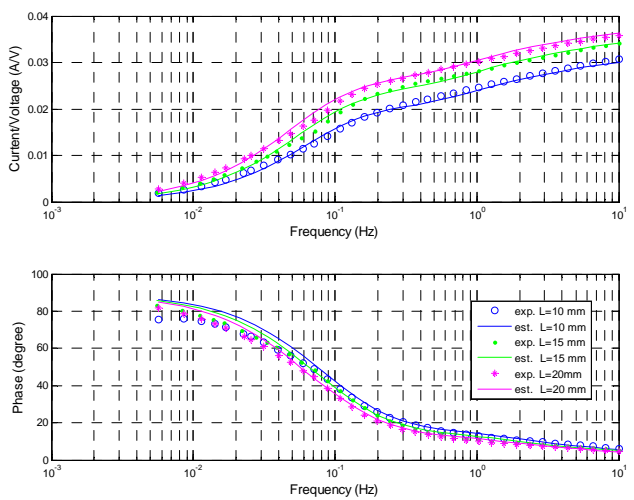

(a) 

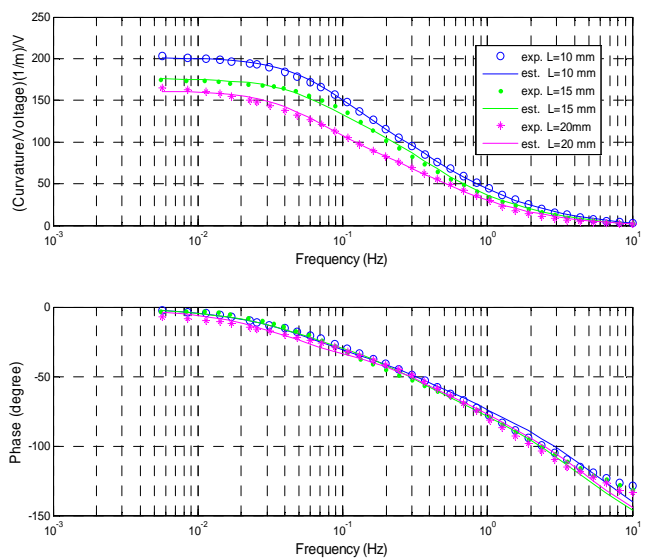

(b)

Fig. 13. Frequency response of electrical admittance (a) and curvature (b) of the actuator at $0.1 \mathrm{~V}$ with different lengths of the actuator
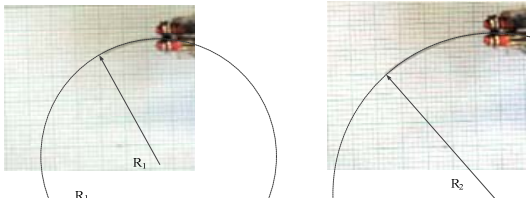

Fig. 14. Deformation of the actuators with different lengths under the same applied voltage.

To verify the proposed model, the dimensions of the actuator were changed and then the experimental results were compared with the simulation results to verify the feasibility of the proposed model. First, the length of actuator was changed to three different values where $L=10$, 15 and $20 \mathrm{~mm}$. The frequency of the electrical admittance at different lengths was measured and estimated using the parameters given above in order to compare it with the experimental results for $0.1 \mathrm{~V}$. The frequency of the electrical admittance and curvature of the proposed model | for different lengthsare shown in Fig. 13Fig. 13 (a) and (b), respectively. The proposed electrical model and the parameters $\left(D\right.$ and $\left.C_{d}\right)$ give a good estimation of the electrical admittance when the length of the actuator is changed. The deformation of the actuators with different | lengths is shown in Fig. 14Fig. 14 where the longer actuator has a larger radius curvature. The curvature of the actuator was also measured and simulated with the proposed model. The three parameters $\mathrm{k}_{0}, \mathrm{k}_{1}$, and $\mathrm{k}_{2}$ were estimated based on a non-linear least square method and are shown in Fig. 15Fig. 15. The results reveal that when the length of the actuator waschanged the parameters of the mechanical properties also changed.

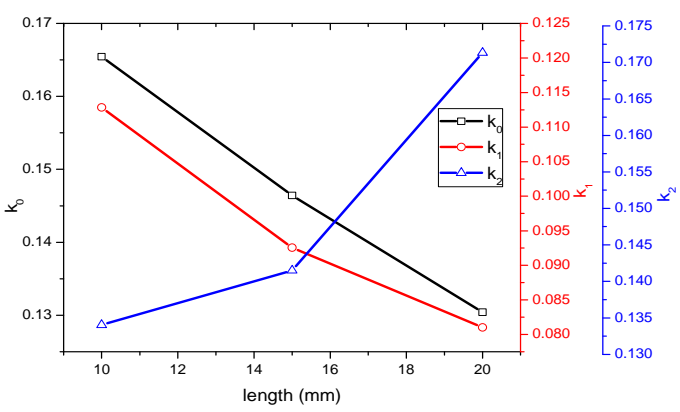

Fig. 15. The variation of $\mathrm{k}_{0}, \mathrm{k}_{1}$ and $\mathrm{k}_{2}$ with respect to the length of the actuator at $0.1 \mathrm{~V}$.

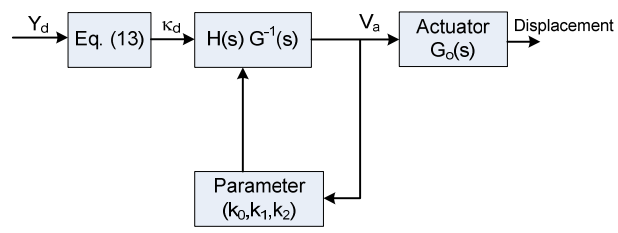

Fig. 16. Schematic of the inversion-based control system

\section{CONTROL OF THE ACTUATOR}

One object of our proposed model is to develop an inversion-based feedforward controller for biomedical applications such as a cochlear implant [4]. The experimental results from the controller will further validate the model and the assumptions made to establish the model in the previous sections. Rewriting Eq. (14) gives:

$$
\kappa_{d}=G(s) V_{a}(s)
$$

by letting:

$$
G(s)=K_{c} \frac{\tanh \left(\sqrt{\frac{2 R_{e}}{Z_{i}(s)} L}\right)}{s \sqrt{2 R_{e} Z_{i}(s)}}
$$

Based on the transfer function in Eq.(18), we can estimate the voltage required for a desired displacement by using the inverse actuator model:

$$
V_{a}=Y_{d} G^{-1}(s)
$$

where $V_{a}$ is an applied voltage, $Y_{d}$ is a desired displacement and the inverse model $G^{-1}(s)$ will compensate for the actuator dynamics. 

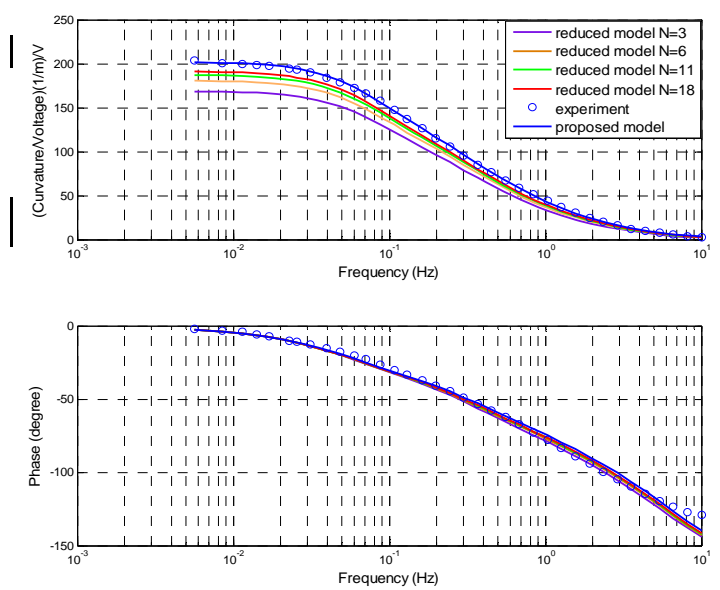

Fig. 17. Comparison of transfer function for different values of $\mathrm{N}$.

The inverted transfer function $G^{-1}(s)$ can be derived by a direct inversion using [5]:

$$
\frac{\tanh \left(\frac{1}{2} \sqrt{\frac{X}{Y}}\right)}{4 \sqrt{X Y}}=\sum_{n=0}^{\infty} \frac{1}{X+\pi^{2}(2 n+1)^{2} Y}
$$

By letting $X=\frac{1}{Z_{i}}$ and $Y=\frac{1}{8 R_{e} L^{2}}$ and by using Eq.(21), the transfer function G(s) in Eq. (19) is obtained as:

|

$$
G(s)=\frac{\sum_{i=0}^{N-1} b_{i} s^{i}}{\sum_{i=0}^{N} a_{i} s^{i}}
$$

Where $b_{i}$, and $a_{i}$ are the parameters which depend on the dimensions, diffusion coefficient, total capacitance and strain-to-charge density ratio described above.

To define the value of $\mathrm{N}$, we changed its value and compared the results of the modelling with the experimental results, as shown in Fig. 17Fig. 17. As shown in this figure, when $\mathrm{N}=11$ and $\mathrm{N}=18$, the reduced models of $G(s)$ match the experimental model and the proposed model quite well, so we chose $\mathrm{N}=11$ to calculate the inverted transfer function, $G^{-1}(s)$.

$G^{-1}(s)$, however, generates an infinite gain at high frequencies because it has a greater number of zeros than poles. Therefore, a low-pass filter $H(s)$ was used to limit the gain at high frequencies and make the system stable [10].

A schematic diagram of the inversion-based control system $\mid$ is shown in Fig. 16Fig. 16. Based on the desired displacement, the applied voltage $V_{a}$ is calculated by After that, this applied voltage $\left(V_{a}\right)$ is also used to adjust parameters $\left(k_{0}, k_{l}\right.$ and $\left.k_{2}\right)$ of $G^{-1}(s)$. Finally, the displacement of the actuator is compared with the desired displacement.

As shown in Fig. 18Fig. 18 (a), with a desired sinusoidal input at $1 \mathrm{~Hz}$, we see that the output displacement fits the desired displacement perfectly, albeit with a small error. performance of the controller is also verified by comparing the response of the output and the desired displacement $y_{d}=2 \sin (2 \pi t)+0.2 \sin (20 \pi \mathrm{t})$ as shown in Fig. 18Fig. 18 (b). Note that the errors are small when the frequency is 1 but they increase when the frequency of the desired displacement is increased to $10 \mathrm{~Hz}$. Possible reasons for these errors are that the vibrational modes of the actuator [28] are neglected in this model and there is a fixed time lag associated with the actuator and control hardware. It must noted that the resonant frequency of the actuator depends on the size of the actuator [28] and for the actuator we considered, it is to be approximately $15 \mathrm{~Hz}$, which is not far from $10 \mathrm{~Hz}$, the maximum frequency of the identified A comparison between the proposed controller and the robust adaptive controller [6] calculated from figure 9 of [6] is shown in Table 2Table 2 . As this table shows, the ratio between the root mean square errors and the amplitude of input of the proposed controller were similar to the adaptive controller with displacement feedback, but the magnitude and frequency of our case is much larger, as shown in Table 2 Table 2. It follows that the proposed model is valid and accurate enough to satisfactorily implement an inversioninversion-based feedforward controller.

The control performance between the inversion-based variable parameters and inversion-based fixed parameter method was also compared and the results are shown in Fig. 19Fig. 19. Here the errors in the proposed method are less than the inversion-based fixed parameters method because in the proposed method, the voltage is controlled based on its magnitude, as shown in Fig. 20Fig. 20.

The performance of the controller is verified by the external noises, as shown in Fig. 21Fig. 21, because when the noises began, the displacement output did not follow the desired input. Also, when the actuator operated over many cycles, the controller performance also degraded though not significantly, as shown in Fig. 22Fig. 22. These are the disadvantages of the proposed controller.

Table 2. Comparison of different controllers.

\begin{tabular}{|c|c|c|}
\hline XIII. Controller & XIV. Desired input & $\begin{array}{c}\text { XV. Root mean } \\
\text { square errors }\end{array}$ \\
\hline $\begin{array}{c}\text { XVI. Adaptive } \\
\text { controller(Fig. } 9 \\
\text { of[6]) }\end{array}$ & $0.5 \sin (\pi t)+0.5 \sin (0.2 \pi t)$ & 0.067 \\
\hline $\begin{array}{c}\text { Proposed controller } \\
\text { (Fig. 17 (b) }\end{array}$ & $2 \sin (2 \pi t)+0.2 \sin (20 \pi t)$ & 0.141 \\
\hline
\end{tabular}

Formatted: Font: $10 \mathrm{pt}$

Formatted: Font: $10 \mathrm{pt}$ 


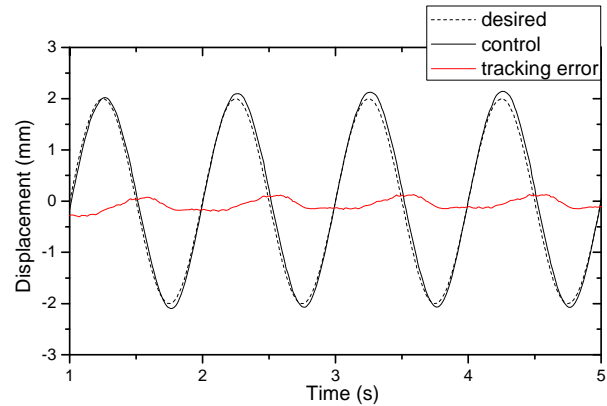

(a)

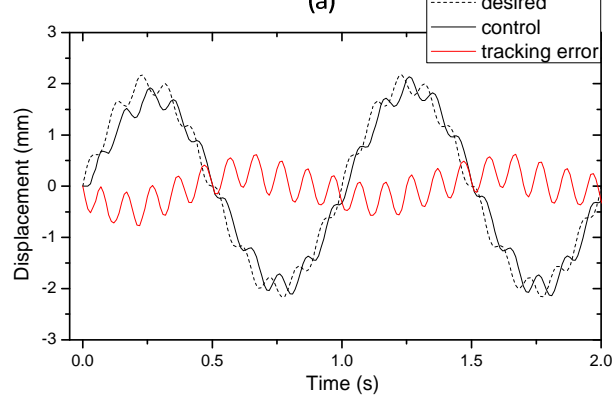

(b)

Fig. 18. Comparison of desired displacement and control results (a) desired sinusoidal wave at $1 \mathrm{~Hz}$ (b) $y_{d}=2 \sin (2 \pi t)+0.2 \sin (20 \pi \mathrm{t})$

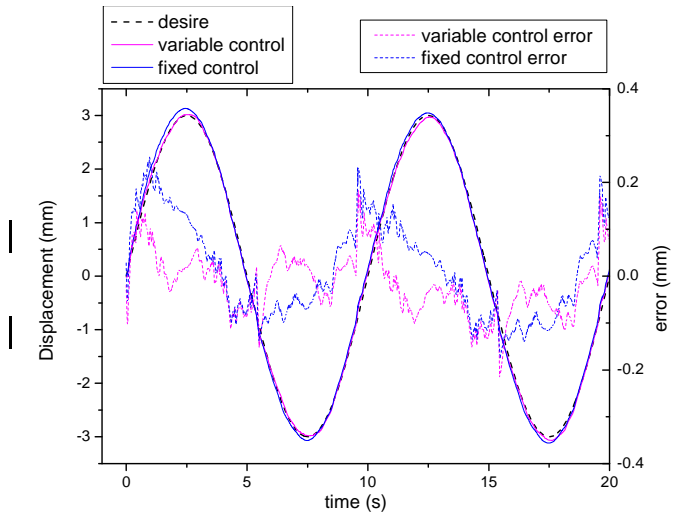

Fig. 19. Comparison between variable and fixed parameter method control method

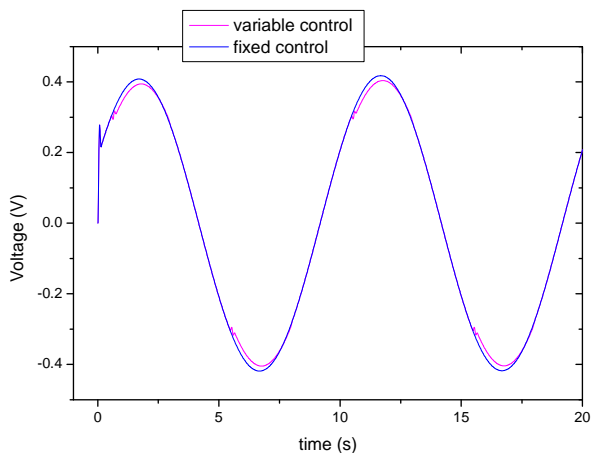

Fig. 20. The applied voltage of two control methods.

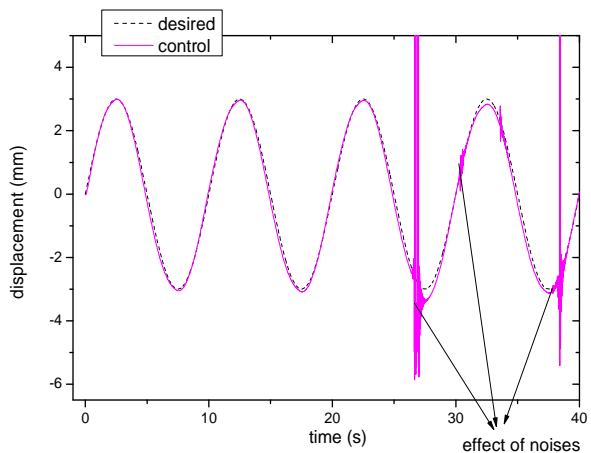

Fig. 21. The effect of noises on the control performance.

The proposed inversion-based controller was also implemented under the ramp and step inputs. The results shown in Fig. 23Fig. 23 and Fig. 24Fig. 24 indicate that the proposed model is accurate enough to track a desired input with very small tracking errors. The voltage applied is shown in Fig. 24Fig. 24, and as with other results, the performance of the inversion control based on the proposed model was quite satisfactory despite some tolerable disturbance and degradation of the actuator [29]. 


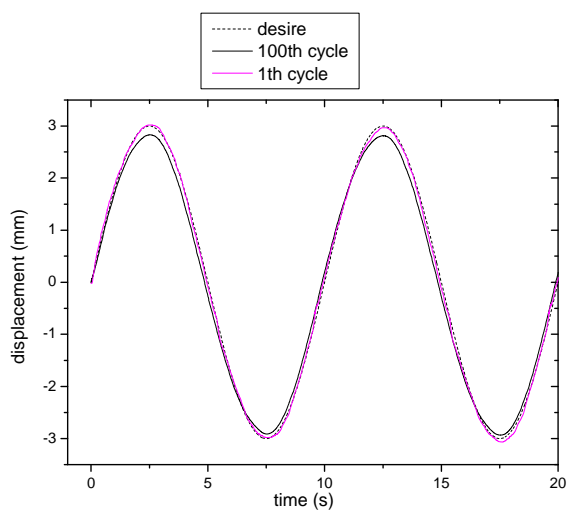

Fig. 22. The repeatable experiment.

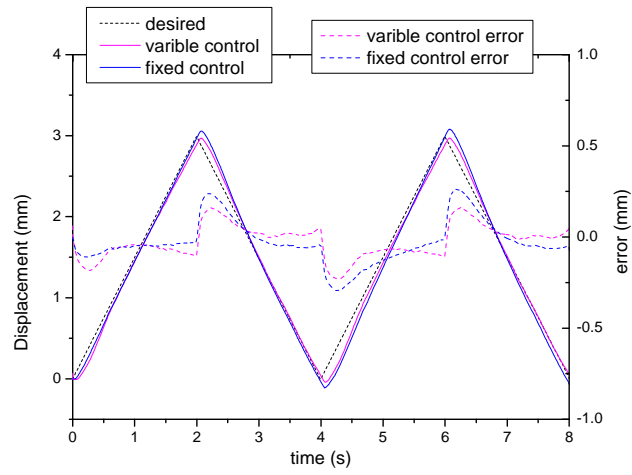

Fig. 23. Comparison of desired and control displacement of ramp input.

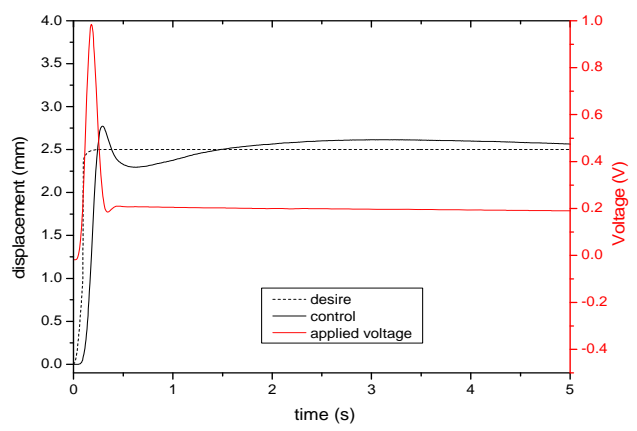

Fig. 24. The control results of step input and applied voltage.

\section{CONCLUSION}

This study has proposed and experimentally validated a new mathematical model to describe the actuator's and has subsequently used this model to implement an inversion-based feedforward controller. The model consists of diffusion impedance connected to the electronic capacitance of an actuator connected in series. The viscoelastic properties of the PPy and PVDF layers were incorporated into the actuator model to describe its mechanical behaviour better. The experimental and theoretical results indicate that the proposed model can accurately predict the behaviour of the electrical admittance spectrum and the displacement of the actuator. The position control results not only validated the model, they also demonstrated the efficacy of the proposed model for a sensorless position control of these actuators.

Future work involves incorporating the non-linear models [24] into the proposed model to describe the 3D behaviour of the actuator.

\section{ACKNOWLEDGMENT}

This work was supported in part by the ARC Centre of Excellence for Electromaterials Science (grant no. CE0561616).

\section{REFERENCES}

11] Y. Bar-Cohen, Electroactive Polymer (EAP) Actuators as Artificial Muscles: Reality, Potential, and Challenges, Second ed., 2004.

[2] G. M. Spinks, G. Alici, S. McGovern, B. Xi, and G. G. Wallace, "Conjugated Polymer Actuators: Fundamentals," in Biomedical Applications of Electroactive Polymer Actuators, ed: John Wiley \& Sons, Ltd, 2009, pp. 193-227.

[3] Y. Wu, G. Alici, G. M. Spinks, and G. G. Wallace, "Fast trilayer polypyrrole bending actuators for high speed applications," Synthetic Metals, vol. 156, pp. 1017-1022, 2006.

[4] D. Zhou, G. G. Wallace, G. M. Spinks, L. Liu, R. Cowan, E. Saunders, and C. Newbold, "Actuators for the cochlear implant," Synthetic Metals, vol. 135-136, pp. 39-40, 2003.

[5] J. D. Madden, "Conducting Polymer Actuators," Ph. D. Thesis Anonymous Cambridge, MA: Massachusetts Institute of Technology, 2000.

[6] F. Yang, T. Xiaobo, and G. Alici, "Robust Adaptive Control of Conjugated Polymer Actuators," Control Systems Technology, IEEE Transactions on. vol. 16, pp. 600-612.2008.

[7] T. Shoa, Y. Dan Sik, K. Walus, and J. D. W. Madden, "A Dynamic Electromechanical Model for Electrochemically Driven Conducting Polymer Actuators," Mechatronics, IEEE/ASME Transactions on, vol. 16, pp. 42-49, 2011.

[8] A. Della Santa, D. De Rossi, and A. Mazzoldi, "Performance and work capacity of a polypyrrole conducting polymer linear actuator," Synthetic Metals, vol. 90, pp. 93-100, 1997.

[9] C. H. Nguyen, G. Alici, and G. G. Wallace, "Modelling trilayer conjugated polymer actuators for their sensorless position control," Sensors and Actuators A: Physical, 2012.

[10]S. W. John, G. Alici, and C. D. Cook, "Inversion-Based Feedforward Control of Polypyrrole Trilayer Bender Actuators," Mechatronics, IEEE/ASME Transactions on, vol. 15, pp. 149-156, 2010.

11]E. Smela, "Conjugated Polymer Actuators for Biomedical Applications," Advanced Materials, vol. 15, pp. 481-494, 2003.

[12]S. Hara, T. Zama, W. Takashima, and K. Kaneto, "Tris(trifluoromethylsulfonyl)methide-doped polypyrrole as a conducting polymer actuator with large electrochemical strain," Synthetic Metals, vol. 156, pp. 351-355, Feb 12006.

[13]Y. Fang, X. Tan, and G. Alici, "Redox level-dependent impedance model for conjugated polymer actuators," Sensors and Actuators B: Chemical, vol. 132, pp. 182-190, 2008.

[14]J. Bobacka, A. Lewenstam, and A. Ivaska, "Electrochemical impedance spectroscopy of oxidized poly(3,4-ethylenedioxythiophene) film electrodes in aqueous solutions," Journal of Electroanalytical Chemistry, vol. 489, pp. 17-27, Jul 282000. 
[15]J. Bisquert, "Theory of the Impedance of Electron Diffusion and Recombination in a Thin Layer," The Journal of Physical Chemistry vol. 106, pp. 325-333, 2001

[16] G. M. Spinks, B. B. Xi, D. Z. Zhou, V. T. Truong, and G. G. Wallace, "Enhanced control and stability of polypyrrole electromechanical actuators," Synthetic Metals, vol. 140, pp. 273-280, Feb 272004.

[17]J. G. A. Madden, "Development and Modeling of Conducting Polymer Actuators and the fabrication of a conducting polymer based feedback loop," Ph. D. Thesis Anonymous Cambridge, MA: Massachusetts Institute of Technology, 2003.

[18] L. E. Malvern, "Introduction to the Mechanics of a Continuous Medium," Englewood Cliffs, NJ: Prentice-Hall, 1969.

[19]T. Pritz, "Unbounded complex modulus of viscoelastic materials and the Kramers-Kronig relations," Journal of Sound and Vibration, vol. 279, pp. 687-697, 2005

[20] J. J. Aklonis, W. J. MacKnight, and M. C. Shen, "Introduction to polymer viscoelasticity," New York, Wiley-Interscience, 1972.

[21]S. W. John, "Modelling and control of conducting polymer actuator," Ph. D thesis, School of Mechnical, Materials and Mechatronic Engineering, University of Wollongong, 2008.

[22] A. Price, T. Gillen, C. Liu, C. O'Shaughnessy, and H. Naguib, "Evaluation of porous membrane core elasticity and porous morphology for polypyrrole trilayer actuators," Journal of Cellular Plastics vol. 48, pp. 25-42, 2012.

[23]Q. Pei and O. Inganaes, "Electrochemical applications of the bending beam method. 1. Mass transport and volume changes in polypyrrole during redox," The Journal of Physical Chemistry, vol. 96, pp. 1050710514, 2011/09/27 1992.

[24]G. Alici, "An effective modelling approach to estimate nonlinear bending behaviour of cantilever type conducting polymer actuators," Sensors and Actuators B: Chemical, vol. 141, pp. 284-292, 2009.

[25] W. Zheng, "Analysis of high performance polypyrrole actuators," Doctor of Philosophy, Department of Chemistry, University of Wollongong, 2011.

[26]Y. Fang, X. Tan, Y. Shen, N. Xi, and G. Alici, "A scalable model for trilayer conjugated polymer actuators and its experimental validation," Materials Science and Engineering: C, vol. 28, pp. 421-428, 2008.

[27]G. Alici, B. Mui, and C. Cook, "Bending modeling and its experimental verification for conducting polymer actuators dedicated to manipulation applications," Sensors and Actuators A: Physical, vol. 126, pp. 396-404, 2006.

[28] S. W. John, G. Alici, and C. D. Cook, "Validation of a Resonant Frequency Model for Polypyrrole Trilayer Actuators", IEEE/ASME Transactions on Mechatronics, Vol.13, No.4, pp.401 -- 409, August 2008.

[29] G. Alici and N. H. Nam, "Performance Quantification of Conducting Polymer Actuators for Real Applications: A Microgripping System," Mechatronics, IEEE/ASME Transactions on, vol. 12, pp. 73-84, 2007.

[30] C.-H. Tso, J. D. Madden, and C. A. Michal, "An NMR study of ions in polypyrrole," Synthetic Metals, vol. 157, pp. 460-466, 2007.

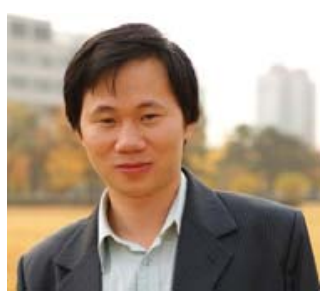

Chuc Huu Nguyen received the B.S degree in Automatic Control engineering from $\mathrm{HCM}$ City

University of Technology, Vietnam in 2003 and the $\mathrm{PhD}$ degree in engineering from School of Mechanical Engineering, Sungkyunkwan University, South Korea, 2009. He is currently an Associate Research Fellow at ARC Associate Research Fellow at ARC
Centre of Excellence for Centre of Excellence for
Electromaterials Science and School of Mechanical, Materials and Mechatronic Engineering, University of Wollongong. His research interests include artificial muscle actuators, sensors and soft robotics.

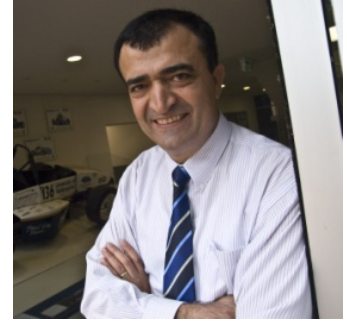

Gursel Alici received a Ph.D. degree in Robotics from the Department of Engineering Science, Oxford University, UK, in 1994. He is currently a Professor at the University of Wollongong, NSW, Australia, where he is the head of School Mechanical, Materials and Mechatronic Engineering. His current research interests include intelligent mechatronic systems involving mechanisms/serial/parallel robot manipulators, macro/micro/nano robotic systems for medical applications, and conducting polymers as macro/micro/nano sized-actuators and sensors for soft robotic and biobio-inspired applications.

He was a technical editor of IEEE/ASME Transactions on Mechatronics during 2008-2012. He is a member of the Mechatronics National Panel formed by the Institution of Engineers, Australia. He has served on the international program committee of numerous IEEE/ASME international conferences on Robotics and Mechatronics. He was the general chair of 2013 IEEE/ASME International Conferences on Advanced Intelligent Mechatronics held in Wollongong, Australia. $\mathrm{He}$ is one of the chief investigators of the ARC Center of Excellence for Electromaterials Science (ACES) in the Energy conversion and Bionics programs. He has published more than 220 refereed publications in his areas of research. Gursel Alici is the recipient of the Outstanding Contributions to Teaching and Learning (OCTAL) award in 2010, and 2013 Vice-Chancellor's Interdisciplinary Research Excellence Award from the University of Wollongong.

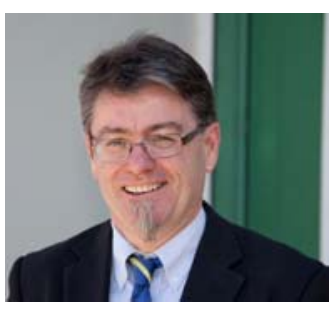

Gordon Wallace obtained his Ph.D. from Deakin University, Geelong, Australia, in 1983. He joined the University of Wollongong in 1986 and is currently Director of the Polymer Research Institute (http://ipri.uow.edu.au/aboutipri/ind ex.html), Executive Research Director of the ARC Centre of Director of the ARC Centre of
Excellence for Electromaterials Excellence for Electromaterials (http://www.electromaterials.edu.au/), and Australian Laureate Fellow. Professor Wallace's research interests include organic conductors, nanomaterials and electrochemical probe methods of analysis, and the use these in the development of Intelligent Polymer Systems. A current focus involves the use of these tools and materials in developing biobio-communications from the molecular to skeletal domains in order to improve human performance via medical bionics.

$\mathrm{He}$ is a fellow of the Australian Academy of Science and the Australian Academy of Technological Sciences and Engineering. He has published over 650 papers with 15,000 citations and h-index of 56, and numerous patents in the areas of organic conductors, nanomaterials, the development of intelligent polymer systems, and their exploitation in medical bionics and energy production and storage. 